\title{
MORTALIDADE DO ÁCARO PREDADOR NEOSEIULUS californicus (ACARI: PHYTOSEIIDAE) EM TESTES DE TOXICIDADE RESIDUAL DE INSETICIDAS E ACARICIDAS USUAIS EM POMÁCEAS ${ }^{1}$
}

\author{
MARCELO GUSTAVORUIZ ${ }^{2} \&$ GILBERTO JOSÉ DE MORAES
}

RESUMO - Os ácaros fitoseídeos, especialmente Neoseiulus californicus (McGregor), são importantes agentes de controle biológico de ácaros tetraniquídeos-praga nas culturas de pomáceas no "Alto Valle del Río Negro y Neuquén”, Argentina. Neste trabalho, avaliou-se a mortalidade de $N$. californicus quando exposto a resíduos dos inseticidas azimphos-methyl, carbaryl e cyfluthrin, e dos acaricidas cyhexatin e propargite. Os produtos foram aplicados às concentrações recomendadas em plantas de pereira. Um, três, seis e dez dias após a aplicação (DAA), folhas tratadas foram retiradas das plantas para a preparação de unidades experimentais. Cinco adultos de $N$. californicus, provenientes de criação-estoque, foram transferidos para cada unidade, onde pólen de taboa foi fornecido como alimento. As unidades foram mantidas a $25 \pm 2{ }^{\circ} \mathrm{C}, 60 \pm 10 \%$ de umidade relativa e fotoperíodo de $14 \mathrm{~h}$. A mortalidade do ácaro foi avaliada $24 \mathrm{~h}$ após o confinamento. As médias de mortalidade foram comparadas pelo teste de Dunnett, a 5\% de probabilidade. A progressão do declínio do efeito dos produtos testados foi submetida à análise de regressão. Nas duas primeiras datas de avaliação, todos os produtos apresentaram valores de mortalidade significativamente diferentes da testemunha tratada com água. Seis dias após a aplicação, propargite, cyhexatin e cyfluthrin apresentaram mortalidade de aproximadamente $30 \%$, enquanto a mortalidade nos tratamentos azimphos-methyl e carbaryl apresentou níveis estatisticamente similares aos da testemunha. Dez dias após a aplicação, a mortalidade em todos os tratamentos não diferiu significativamente da testemunha. O efeito de todos os produtos apresentou declínio progressivo ao longo do período de observação, sendo significativa a $1 \%$ de probabilidade a regressão linear negativa para os valores obtidos. Os maiores efeitos negativos sobre a sobrevivência de $N$. californicus corresponderam aos acaricidas testados. Azimphos-methyl foi o produto que menos afetou a sobrevivência do ácaro predador. Os inseticidas testados, usados na região do “Alto Valle del Río Negro y Neuquén” para o controle de Cydia pomonella, praga-chave das culturas de pomáceas, apresentaram baixa toxicidade sobre $N$. californicus.

Termos para indexação: Controle biológico - Manejo Integrado de Pragas - Seletividade - Pomáceas.

\section{MORTALITY OF THE PREDATORY MITE NEOSEIULUS californicus (ACARI: PHYTOSEIIDAE) IN RESIDUAL TOXICITY PERSISTENCE TESTS}

\begin{abstract}
Phytoseiid mites, mainly Neoseiulus californicus (McGregor), are important biological control agents of Tetranychidae pest mites in pip fruit crops in the region known as "Alto Valle del Río Negro y Neuquén", Argentina. We assessed the mortality of $N$. californicus when exposed to residues of the insecticides azimphos-methyl, carbaryl and cyfluthrin, as well as the acaricides cyhexatin and propargite. Pear plants were sprayed up to dip-point with pesticides in their recommended label concentrations. One, 3, 6 and 10 days after application (DAA), leaves were collected from treated plants and used to establish experimental arenas. Five adult laboratoryreared $N$. californicus specimens were transferred into each arena which contained Southern cattail pollen as food source. Experimental arenas were kept at $25 \pm 2{ }^{\circ} \mathrm{C}, 60 \pm 10 \% \mathrm{RH}$ and a photoperiod of 14 hours. Mite mortality was assessed 24 hours after the confinement. The completely randomized design was adopted for data statistical analysis, mortality means were compared by Dunnett's test ( $\mathrm{p}<$ 0.05). Progression of pesticide's effect decline was submitted to regression analysis. On 1 and 3 DAA mean mortality in all of the treatments was significantly different from that of the water-treated control. On the sixth DAA, propargite, cyhexatin and cyfluthrin treatments caused about 30\% mortality, while mortality levels in treatments with azimphos-methyl and carbaryl were statistically similar to that of control treatment. On the tenth DAA, mortality in none of the pesticide treatments differed from that of control. All of the pesticide treatments presented progressive decline throughout the experimental period, being significant $(\mathrm{p}<0.01)$ the negative linear regression obtained. Tested acaricides produced the greatest negative effects on the survival of $N$. californicus. Azimphosmethyl was the pesticide that least affected the survival of the predatory mite. Tested insecticides, used in the "Alto Valle del Río Negro y Neuquén" region to control codling moth, C. pomonella, key pest of pip fruit crops, showed low toxicity to N. californicus. Index Terms: Biological Control, Integrated Pest Management, Selectivity, Pip fruit.
\end{abstract}

'(Trabalho 273-07). Recebido em: 21-11-2007. Aceito para publicação em: 12-08-2008.

${ }^{2}$ Pós-graduando em Agronomia - Entomologia Agrícola FCAV-UNESP. Endereço atual: Facultad de Ciencias Agrarias - Universidad Nacional del Comahue. C.C. 85 - (8303) Cinco Saltos, Río Negro, Argentina. marcelorzar@yahoo.com.ar

${ }^{3}$ Professor Doutor. Departamento de Entomologia, Fitopatologia e Zoologia Agrícola. ESALQ-USP. 13418-900 Piracicaba, SP, Brasil. gjmoraes@esalq.usp.br 


\section{INTRODUÇÃO}

Cydia pomonella L. (Lepidoptera: Tortricidae), conhecida como broca-da-maçã no Brasil e como "carpocapsa" em vários países de língua espanhola, é a praga-chave das culturas de pêra e maçã na região do "Alto Valle del Río Negro y Neuquén", na Argentina. Inseticidas dos grupos organofosforados, carbamatos e piretróides são utilizados para o seu controle, sendo realizadas de seis a doze pulverizações desses produtos por ciclo produtivo da cultura.

$\mathrm{O}$ aumento das populações de pragas secundárias, tais como os ácaros Panonychus ulmi (Koch) e Tetranychus urticae Koch (Acari: Tetranichydae), parece estar relacionado ao uso intensivo desses produtos, isso devido, em parte, ao efeito negativo daqueles produtos sobre os inimigos naturais daqueles ácaros (Cichón et al., 1994, 1996).

Nas culturas de pomáceas, o controle biológico exercido pelos ácaros predadores fitoseídeos (Acari: Phytoseiidae) é capaz de manter as populações de ácaros fitófagos em níveis que não produzem danos econômicos (Blommers, 1994; Monteiro, 1994, 2002; Gerson et al., 2003). Tem-se demonstrado que em cada região pode haver diferentes espécies de Phytoseiidae e que, antes de programas de manejo integtrado de pragas serem iniciados, a diversidade da fauna local deve ser estudada para se determinar o potencial de controle exercido pelos predadores que já ocorrem na região (Gerson et al., 2003; Blommers, 1994; Costa-Comelles et al., 1994). Na região do "Alto Valle del Río Negro y Neuquén”, são reconhecidas as necessidades de se contar com um programa de manejo integrado de pragas e da utilização de produtos seletivos nas culturas de frutíferas (Cichón et al., 1996, 1994), permitindo a ação benéfica de ácaros predadores, de maneira particular Neoseiulus californicus (McGregor), que é o principal inimigo natural encontrado em campo, nessas culturas (Cichón et al., 1996, 1994; Müther, 1998; Ruiz et al., 2005).

Na Argentina, a legislação estabelece a obrigatoriedade do controle de $C$. pomonella em todas as culturas consideradas hospedeiras daquela praga, tendo sido criado para isso o "Programa Nacional de Supresión de la Carpocapsa" (PNSC). O PNSC tem impulsionado várias medidas, entre as quais se encontram o uso da técnica de confusão sexual, a erradicação de pomares abandonados ou em risco fitossanitário, e o financiamento da aquisição do inseticida azimphos-methyl para os produtores economicamente menos favorecidos. Muitos agricultores, entretanto, vão além disso, fazendo uso de outros agrotóxicos na cultura. Nessa situação, é imprescindível o conhecimento dos possíveis efeitos dos agrotóxicos sobre as populações dos ácaros fitoseídeos.

A avaliação dos efeitos dos agrotóxicos sobre os organismos benéficos requer a consideração de vários fatores relacionados. Por isso, pesquisadores da "I.O.B.C. - W.P.R.S. (Internacional Organization for Biological Control/ West Palaeartic Region Section)" desenvolveram uma estratégia de avaliação mundialmente reconhecida, baseada numa seqüência de testes padronizados de laboratório, semicampo e campo, que permite a determinação dos efeitos colaterais daquelas substâncias (Hassan et al., 1987; 1994).

Os testes de persistência são bioensaios que objetivam avaliar os efeitos produzidos sobre espécies benéficas, pelo seu contato com os depósitos de agrotóxicos presentes num substrato como consequiência de tratamentos fitossanitários. O princípio básico desses testes é submeter os organismos ao contato forçado com depósitos de agrotóxicos expostos aos fatores de degradação durante períodos de tempo previamente estabelecidos (Hassan et al., 1994; Vanwetswinkel \& Plevoets, 1985).

O objetivo deste trabalho foi avaliar a persistência do efeito de alguns inseticidas e acaricidas comumente usados em pomares de pomáceas na região do "Alto Valle del Río Negro y Neuquén", na sobrevivência de $N$. californicus, com o fim de estimar o possível impacto do uso desses produtos sobre o controle natural de ácaros fitófagos.

\section{MATERIAL E MÉTODOS}

No experimento, o protocolo para os testes de persistência estabelecido pela I.O.B.C./W.P.R.S. (Hassan et al., 1994; Vanwetswinkel \& Plevoets, 1985) foi adoptado quase completamente, porém apenas a mortalidade de $N$. californicus, quando exposto aos resíduos dos produtos testados, foi registrada. O efeito dos produtos na oviposição não foi avaliado.

Para a realização deste trabalho, foram utilizados exemplares de $N$. californicus obtidos de uma criação-estoque mantida na "Facultad de Ciencias Agrarias da Universidad Nacional del Comahue" (FCA-UNCo), Cinco Saltos, Río Negro, Argentina, segundo a método de McMurtry \& Scriven (1965), que constou basicamente de bandejas isoladas por água contendo os predadores, que foram alimentados com pólen de taboa (Typha domingensis Pers.). Foram usadas unidades de criação correspondentes a uma camada de espuma sintética sobre a qual se colocou uma lâmina de plástico de cor preta. $\mathrm{O}$ conjunto foi circundado com uma faixa de algodão hidrófilo umedecido para o confinamento dos ácaros, introduzido em bandejas plásticas $(6 \times 22 \times 14,5 \mathrm{~cm})$. As bandejas foram mantidas em sala climatizada a $25 \pm 2{ }^{\circ} \mathrm{C}, 60 \pm 10 \%$ de umidade relativa e fotoperíodo de $14 \mathrm{~h}$ (Ruiz et al., 2001).

Essa colônia havia sido iniciada cerca de três meses antes do início deste trabalho, com exemplares coletados num pomar de macieiras pertencente à FCA-UNCo, o qual não tinha recebido tratamentos químicos nas duas temporadas anteriores. Exemplares coletados aleatoriamente da colônia foram montados em lâminas com meio de Hoyer para a confirmação de identificações antes do início do trabalho.

Testaram-se os efeitos de 3 inseticidas comumente utilizados para o controle de C. pomonella e 2 acaricidas comumente utilizados para o controle de tetraniquídeos na região considerada, os quais foram aplicados nas concentrações recomendadas em pomáceas (Tabela 1).

No mês de março de 2002, 18 plantas de pereira, Pyrus communis L., cultivar William's, foram selecionadas em um pomar de seis anos de idade, com espaçamento de 3,5 m x 3,0 m, no 
município de Cinco Saltos (3856`S, 6759`W, altitude $285 \mathrm{~m}$ ), localizado no "Alto Valle del Río Negro y Neuquén”. As plantas escolhidas eram homogêneas quanto ao tamanho, estado de desenvolvimento e condições gerais. O pomar não havia recebido tratamentos químicos durante, pelo menos, um ano antes do início do trabalho.

Cada produto foi aplicado em três plantas tomadas dentre aquelas pré-selecionadas. Como testemunha, três plantas foram pulverizadas com água. A aplicação de cada produto (assim como da água, no tratamento-testemunha) foi feita até o ponto de escorrimento, com o uso de uma pistola, à pressão de 200 psi $(1379 \mathrm{kPa})$, fornecida por turboatomizador acoplado a um trator. As plantas pulverizadas eram separadas umas das outras por, pelo menos, três plantas em toda direção, para limitar possíveis efeitos indesejáveis causados pela deriva no momento da aplicação. A aplicação dos produtos foi realizada no final da tarde (19:00), com $23{ }^{\circ} \mathrm{C}$, sem vento. Os valores médios de temperatura e umidade relativa no período em que se desenvolveu o experimento, foram $16,2^{\circ} \mathrm{Ce} 63,9 \%$, respectivamente. Não houve precipitação durante todo o período. Os registros foram obtidos junto à estação meteorológica da FCA-UNCo, distante aproximadamente $2,5 \mathrm{~km}$ do pomar em que o experimento foi realizado.

Um, três, seis e dez dias após o tratamento, foram retiradas três folhas do terço médio de cada uma das plantas tratadas. As folhas foram tomadas ao acaso, evitando-se, porém, aquelas defeituosas. Para retirá-las das plantas, o pecíolo de cada folha foi segurado com uma pinça, cortando-se sua extremidade distal com uma tesoura. Em seguida, as folhas foram acondicionadas em um saco de papel, evitando-se o contato com as mãos do operador.

Os sacos contendo as folhas foram imediatamente acondicionados em sacos plásticos, os quais foram colocados dentro de uma caixa térmica $(50 \times 25 \times 35 \mathrm{~cm})$ contendo $2,5 \mathrm{dm}^{3} \mathrm{de}$ gelo para o seu transporte até o laboratório na FCA-UNCo, onde foram mantidas em geladeira a $6^{\circ} \mathrm{C}$ até o momento da sua utilização dentro das $12 \mathrm{~h}$ subseqüentes.

No laboratório, unidades experimentais de $3 \mathrm{~cm}$ de diâmetro com as folhas coletadas foram confeccionadas. Para isso, colocou-se uma camada de papel-filtro dentro de uma tampa invertida de placa de Petri de $15 \mathrm{~cm}$ de diâmetro; sobre essa camada, colocou-se uma das folhas coletadas, com a face superior voltada para cima. Sobre aquela folha, colocou-se uma lâmina de plástico com um orifício circular de $3 \mathrm{~cm}$ de diâmetro, vedandose o espaço entre a margem do orifício e a superfície da folha com adesivo do tipo "tangle-foot" para impedir a fuga dos ácaros.

Sob microscópio estereoscópico e com auxílio de pincel, cinco adultos de $N$. californicus, provenientes da criaçãoestoque, foram transferidos para cada unidade. Nas primeiras 3 datas de avaliação, os adultos utilizados foram exclusivamente fêmeas. Devido a problemas na criação-estoque, na quarta avaliação, foi utilizado $20 \%$ de machos, repartidos homogeneamente entre os tratamentos. Uma pequena quantidade de pólen de taboa foi introduzida em cada unidade como fonte de alimento para os predadores. As unidades foram mantidas em sala climatizada a $25 \pm 2{ }^{\circ} \mathrm{C}, 60 \pm 10 \%$ de umidade relativa e fotoperíodo de $14 \mathrm{~h}$.

Em todas as datas, avaliou-se a mortalidade dos ácaros, em cada unidade, vinte e quatro horas após a transferência dos predadores. Considerou-se morto o ácaro que não apresentou qualquer movimento quando tocado levemente com um pincel.

Para a comparação estatística das médias de mortalidade, adotou-se o delineamento inteiramente casualizado, com seis tratamentos e seis repetições, sendo realizadas análises separadas para cada data de coleta das folhas no campo. As análises de variância foram realizadas utilizando o programa Estat da Faculdade de Ciências Agrárias e Veterinárias (FCAV) - UNESP (ESTAT, 2004) e as comparações de médias, pelo teste de Dunnett, a 5\% de probabilidade, utilizando o programa SAS (SAS, 2000). Os dados foram previamente transformados em [arco-seno (raiz quadrada de $\mathrm{x}$ )]. Para analisar o declínio do efeito dos produtos testados, as porcentagens de mortalidade nos distintos períodos, para cada produto, foram relacionadas através de regressões feitas com o programa Estat da FCAV - UNESP.

\section{RESULTADOS}

Em todos os períodos de avaliação, a mortalidade no tratamento-testemunha foi sempre muito reduzida, atingindo no máximo 3,3\% nas avaliações correspondentes aos seis e dez dias após a aplicação dos produtos (Tabela 2).

No primeiro dia após a pulverização das plantas, todos os produtos avaliados apresentaram efeito negativo na sobrevivência de $N$. californicus em relação à testemunha (Tabela 2). A mortalidade registrada nos tratamentos com os acaricidas cyhexatin e propargite foi de 90 e $63 \%$, respectivamente. Dentre os inseticidas, carbaryl e cyfluthrin causaram mortalidade de aproximadamente 50\%, e azimphos-methyl, de $30 \%$.

$\mathrm{Na}$ segunda avaliação, ambos os acaricidas apresentaram mortalidade próxima a $50 \%$, enquanto para todos os inseticidas, a mortalidade média atingiu valores próximos a $20 \%$.

No sexto dia após a pulverização, propargite, cyhexatin e cyfluthrin continuaram a apresentar efeito negativo na sobrevivência de $N$. californicus, com níveis de mortalidade próximos a $30 \%$. Entretanto, azimphos-methyl e carbaryl mostraram-se estatisticamente similares à testemunha. No décimo dia após a pulverização, nenhum tratamento diferiu significativamente da testemunha.

O estudo da relação entre os efeitos dos produtos e o tempo desde a aplicação mostrou a existência de regressões lineares negativas significativas ( $\mathrm{p}<0,01$ ) (Figura 1), observandose correlação entre o tempo e o efeito residual de cada produto. Os coeficientes de regressão $\left(\mathrm{R}^{2}\right)$ variaram entre 71 e $98 \%$.

Segundo as classes propostas pela IOBC para a avaliaçao da duração do efeito dos agrotóxicos (Hassan et al., 1987), os produtos testados podem ser considerados levemente persistentes, já que a duração da sua atividade nociva foi inferior a 15 dias. A taxa de decréscimo do efeito foi mais pronunciada para os dois produtos que se mostraram mais tóxicos ao predador no início (cyhexatin e propargite), sendo esta mais uniforme e menor para os demais produtos testados (Figura 1). 


\section{DISCUSSÃO}

Em uma análise geral, verifica-se que os acaricidas apresentaram efeitos mais pronunciados sobre a sobrevivência de $N$. californicus do que os inseticidas testados. Azimphosmethyl, em particular, foi o produto de menor toxicidade sobre o ácaro predador.

Os resultados indicaram que os inseticidas usados na região do "Alto Valle del Río Negro y Neuquén", para o controle de C. pomonella, apresentaram baixa toxicidade residual sobre N. californicus.

Nos anos 50 e 60, produtos organofosforados e carbamatos eram utilizados tanto como inseticidas quanto como acaricidas, mas posteriormente perderam capacidade de controlar ácaros-praga devido ao desenvolvimento de populações resistentes a vários desses produtos (Morris \& Gonzalez Pape, 1968). Atualmente, o controle de ácaros-praga (especialmente tetraniquídeos) é realizado com acaricidas específicos (Cichón et al., 1996, 1994).

A capacidade dos ácaros fitoseídeos para desenvolver linhagens resistentes aos agrotóxicos tem sido estabelecida em várias oportunidades; essa característica foi um dos principais fatores que levaram ao estabelecimento dos primeiros programas de MIP em macieiras (Croft, 1975; Croft \& Mc Groarty, 1977). No caso de $N$. californicus, sua capacidade de resistir aos efeitos dos organofosforados foi reportada na América do Sul três décadas atrás (Croft et al., 1976). Na região do "Alto Valle de Río Negro y Neuquén”, Müther (1998) destacou a impossibilidade de usar $N$. californicus como espécie bioindicadora, devido a sua insensibilidade aos agrotóxicos de amplo espectro de ação. Recentemente, Poletti (2007) observou que uma população brasileira de $N$. californicus apresentou menor suscetibilidade do que uma população de Phytoseiulus macropilis à ação de vários agrotóxicos utilizados em cultivo protegido. Por sua vez, Silva \& Oliveira $(2006,2007)$ reportaram que $N$. californicus mostrou sensibilidade diferenciada quando exposto à pulverização direta e toxicidade residual de vários agrotóxicos utilizados em citros no Brasil.

Os inseticidas azimphos-methyl e carbaryl começaram a ser utilizados para o controle de pragas nas culturas de pomáceas, na região do "Alto Valle de Río Negro y Neuquén”, nos anos 60, sendo carbaryl também utilizado pelas suas propriedades como raleador químico de frutos. Cyfluthrin, assim como outros vários produtos pertencentes ao mesmo grupo dos piretróides foram usados amplamente nos anos 80. A partir daí, seu uso foi restringido, tendo em vista o seu largo espectro de ação e o conseqüente efeito indesejável sobre organismos benéficos. Os acaricidas testados têm sido utilizados em pomáceas desde os anos 70 .

Neste contexto, tanto a existência em nível de campo de um processo de seleção de populações de Phytoseiidae com sensibilidade reduzida à ação desses produtos, quanto a ocorrência de um processo de insensibilidade intrínseca não podem ser descartadas.

Os acaricidas cyhexatin e propargite apresentam efeito mais prolongado do que os inseticidas testados.

TABELA 1- Detalhes dos produtos considerados no teste de toxicidade residual. Cinco Saltos, 2002.

\begin{tabular}{|c|c|c|c|c|}
\hline Ingrediente ativo & Marca Comercial & Classe & Grupo Químico & Concentração $^{(1)}$ \\
\hline azimphos-methyl & Gusation SC 36 & Inseticida & Organo-fosforado & 100 \\
\hline carbaryl & Sevin PM 85 & Inseticida & Carbamato & 120 \\
\hline cyfluthrin & Baytroid SC 5 & Inseticida & Piretróide & 30 \\
\hline cyhexatin & Sipcatin SC 50 & Acaricida & Organo-estânico & 30 \\
\hline propargite & Omite PM 30 & Acaricida & Sulfito de alquila & 200 \\
\hline
\end{tabular}

(1) ( $m l$ ou $g$ p.c./ 100 L), p.c.: produto comercial; SC: suspensão concentrada; PM: pó molhável.

TABELA 2- Porcentagem de mortalidade de Neoseiulus californicus em testes de toxicidade residual, realizados em diferentes períodos, após a aplicação dos produtos no campo (DAA: dias após o tratamento), em macieiras. Cinco Saltos, 2002.

\begin{tabular}{|c|c|c|c|c|c|c|c|c|}
\hline \multirow[t]{2}{*}{ Tratamento } & \multicolumn{2}{|l|}{$\mathrm{DAA}=1$} & \multicolumn{2}{|c|}{$\mathrm{DAA}=3$} & \multicolumn{2}{|c|}{$\mathrm{DAA}=6$} & \multicolumn{2}{|l|}{$\begin{array}{l}\mathrm{DAA}= \\
10\end{array}$} \\
\hline & Média ${ }^{1}$ & E.P. $^{2}$ & Média & E.P. & Média & E.P. & Média & E.P. \\
\hline azimphos-methyl & $30,0 *$ & 4,5 & $16,7 *$ & 3,3 & 23,3 & 6,25 & 3,3 & 3,3 \\
\hline carbaryl & $56,7 *$ & 9,6 & $20,0 *$ & 5,2 & 20,0 & 5,26 & 10,0 & 4,7 \\
\hline cyfluthrin & $53,3 *$ & 6,7 & $23,3 *$ & 6,1 & $30,0 *$ & 8,6 & 13,3 & 4,2 \\
\hline cyhexatin & $90,0 *$ & 6,9 & $46,7 *$ & 6,6 & $30,0 *$ & 4,7 & 20,0 & 8,9 \\
\hline propargite & $63,3 *$ & 6,2 & $53,3 *$ & 11,2 & $36,7 *$ & 10,8 & 10,0 & 4,5 \\
\hline testemunha & 0,0 & 0,0 & 0,0 & 0,0 & 3,3 & 2,3 & 3,3 & 2,3 \\
\hline
\end{tabular}

${ }^{1}$ Média: porcentagem média de mortalidade; ${ }^{2}$ E.P.: erro-padrão da média; na mesma data de avaliação, tratamentos seguidos de asterisco diferem estatisticamente da testemunha $(\mathrm{p}<0,05)$, pelo Teste de Dunnett. 


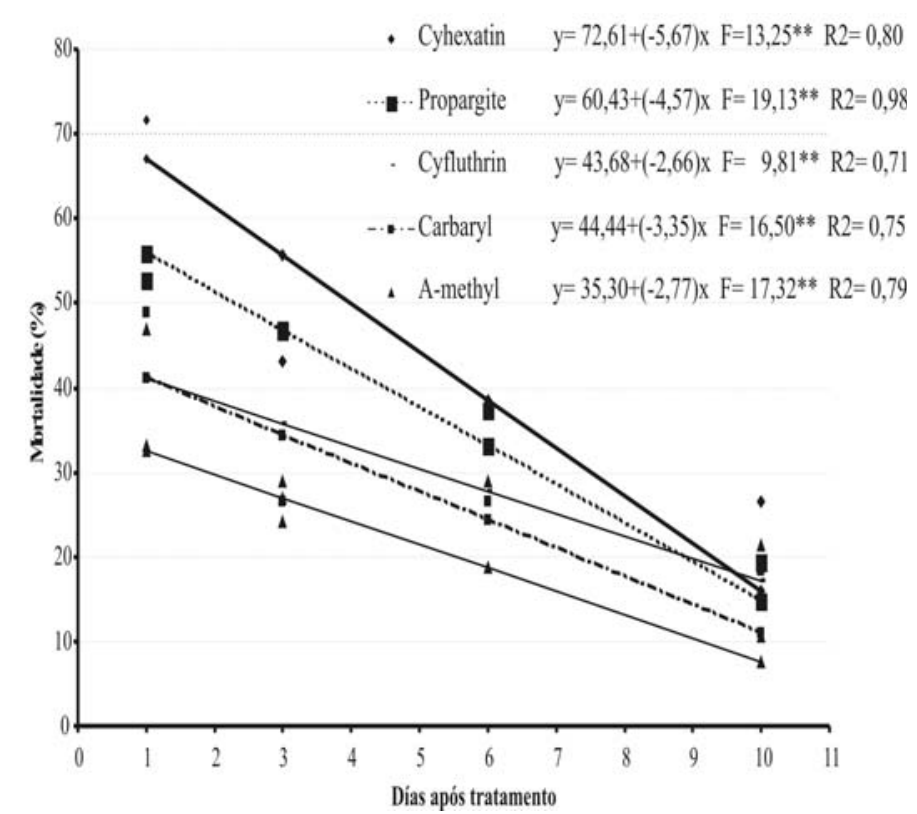

FIGURA 1- Mortalidade de $N$. californicus no teste de toxicidade residual de diferentes produtos químicos. Para cada agrotóxico, os símbolos na reta de regressão indicam os valores esperados; os símbolos fora de linha mostram os valores da porcentagem de mortalidade média registrados no experimento. Cinco Saltos, 2002.

\section{CONCLUSÕES}

1-Os inseticidas azimphos-methyl e carbaryl apresentam baixa toxicidade residual sobre $N$. californicus, nas culturas de pomáceas.

2-Os acaricidas cyhexatin e propargite apresentam efeito mais prolongado do que os inseticidas testados.

\section{REFERÊNCIAS}

BLOMMERS, L.H.M. Integrated pest management in European apple orchards. Annual Review of Entomology, Stanford, v.39: p. 213-241, 1994.

CICHÓN, L.I.; Di MASSI, S.N.; FERNANDEZ, D.E.; MAGDALENA, C.; RIAL, E.J.; ROSSINI, M.N. Guía ilustrada para monitoreo de plagas en frutales de pepita. Buenos Aires: INTA, $1996.73 \mathrm{p}$.

CICHÓN, L.I.; FERNANDEZ, D.E.; MAGDALENA, C. Guía ilustrada para monitoreo de plagas en frutales de pepita. Buenos Aires: INTA, 1994. 52 p.

COSTA-COMELLES, J.; DEL RIVERO, J.M.; FERRAGUT, F.; GARCIA-MARI, F. Control integrado de ácaros en manzano en España. INIA Investigación Agraria, Madrid, Fuera de Serie n.2, p. 49-63, 1994.
CROFT, B.A. Integrated control of apple mites. East Lansing: Michigan State University, 1975. 11 p. (Bulletin, E825)

CROFT, B.A.; MCGROARTY, D.L. The role of Amblyseius fallacis (Acarina: Phytoseiidae) in Michigan apple orchards. East Lansing, Michigan: State University, 197722 p. (Research Report 333)

ESTAT. Sistema para análises estatísticas (V. 2.0). Jaboticabal: Departamento de Ciências Exatas, Faculdade de Ciências Agrárias e Veterinárias, Universidade Estadual Paulista, Jaboticabal, 1994.

GERSON, U.; SMILEY, R.L.; OCHOA, R. Mites (Acari) for pest control. London: Blackwell Science, 2003. 425 p.

HASSAN, S.A.; ALBERT, R.; BIGLER, F.; BLAISINGER, P.; BOGENSCHÜTZ, H.; BOLLER, E.; BRUN, J.; CHIVERTON, P.; EDWARDS, P.; ENGLERT, W.D.; HUANG, P.; INGLESFIELD, C.; NATON, E.; OOMEN, P.A.; OVERMEER, W.P.J.; RIECKMANN, W.; SAMSØE-PETERSEN, L.; STÄUBLI, A.; TUSET, J.J.; VIGGIANI, G.; VANWETSWINKEL, G. Results of the third joint pesticide testing programme by the IOBC/WPRS working group "pesticides and beneficial organisms". Journal of Applied Entomology, Hamburg, v. 103, n. 1, p. $92-107,1987$.

HASSAN, S.A.; BIGLER, F.; BOGENSCHÜTZ, H.; BOLLER,E.; BRUN, J.; CALIS, J.N.M.; COREMANS-PELSENEER, J.; DUSO, C.; GROVE, A.; HEIMBACH, U.; HELYER, N.; HOKKANEN, H.; LEWIS, G.B.; MANSOUR, F.; MORETH, L.; POLGAR, L.; SAMSØE-PETERSEN, L.; SAUPHANOR, B.; STÄUBLI, A.; STERK, G.; VAINIO, A.; van de VEIRE, M.; VIGGIANI, G.; VOGT, $\mathrm{H}$. Results of the sixth joint pesticide testing programme of the IOBC/WPRS working group "pesticides and beneficial organisms”. Entomophaga, Paris, v. 39, n. 1, p. 107-119, 1994.

McMURTRY, J. A.; SCRIVEN, G. T. Insectary production of phytoseiid mites. Journal of Economic Entomology, Lanham, v. 58, n. 2, p. 282-284, 1965.

MONTEIRO, L. B. Manejo integrado de P. ulmi em macieira. Primeiras experiências com a introdução de $N$. californicus. Revista Brasileira de Fruticultura, Jaboticabal, v16, n.1, p.4653, 1994.

MONTEIRO, L. B. Manejo integrado de pragas em macieira no Rio Grande do Sul II. Uso de $N$. californicus para o controle de $P$. ulmi. Revista Brasileira de Fruticultura, Jaboticabal, v. 24, n. 2, p. 395-405, 2002.

MORRIS, G. L.; GONZALEZ PAPE, O. Ácaros del Alto Valle de Río Negro y Neuquén, su control. Buenos Aires, INTA, 1968, 49 p.

MÜTHER, J. Auswirkungen von Pflanzenschutzmitteln und Anbauverfahren auf die Biologie und Ökologie natürlicher Gegenspieler von Spinnmilben - Eine Beurteilung für 
Kernobstbau in Nord-Patagonien, Argentinien. Plits, Stuttgart, v. 16, n. 7 , p. 1-168, 1998.

POLETTI, M. Integração das estratégias de controle químico e biológico para a conservação e liberação dos ácaros predadores Neoseiulus californicus (McGregor) e Phytoseiulus longipes (Banks) (Acari: Phytoseiidae) em programas de manejo do ácaro rajado, Tetranychus urticae Koch (Acari: Tetranychidae). 2007. Tese (Doutorado, área de concentração Entomologia) - Escola Superior de Agricultura Luiz de Queiroz, Universidade de São Paulo, Piracicaba, 2007.

RUIZ, M.G.; GÓMEZ, D.; SEPÚLVEDA, P.; FERNANDES, O.A.; BUSOLI, A.C. Laboratory rearing method of Neoseiulus californicus (McGregor) (Acari: Phytoseiidae) for pesticide selectivity tests. In: SIMPÓSIO DE CONTROLE BIOLÓGICO, 7., 2001, Poços de Calda. Anais... p. 289.

RUIZ, M.G.; LOFEGO, A.C.; MORAES, G.J. de; SOSA, D.; SPERANZA, C.; FERNANDES, O.A. Phytoseiid mites (Acari: Phytoseiidae) from apple trees in Río Negro, Argentina. Spanish Journal of Agricultural Research. Madrid, v. 3, n. 4, p. 437-438, 2005.
SAS INSTITUTE INC. SAS/STAT: user's guide version 8. Cary, NC, 2000.

SILVA, M.Z. da; OLIVEIRA, C.A.L. de. Seletividade de alguns agrotóxicos em uso na citricultura ao ácaro predador Neoseiulus californicus (McGregor) (Acari: Phytoseiidae). Revista Brasileira de Fruticultura, Jaboticabal, v. 28, n. 2, p. 205-208, 2006.

SILVA, M. Z. da; OLIVEIRA, C. A. L. de. Toxicidade residual de alguns agrotóxicos recomendados na citricultura sobre Neoseiulus californicus (McGregor) (Acari: Phytoseiidae). Revista Brasileira de Fruticultura, Jaboticabal, v. 29, n. 1, p. 085-090, 2007.

VANWETSWINKEL, G.; PLEVOETS, P. Standard method to test pesticide side-effects on Amblyseius (Typhlodromus) finlandicus (Oudm.) (Acari: Phytoseiidae) on apples, pears or cherries. In: HASSAN, S.A. (Ed.) Standard methods to test the side-effects of pesticides on natural enemies of insects and mites. Bulletin OEPP/EPPO, Paris, v.15, p.214-255, 1985. 